\title{
Constraints on the Extremely High-Energy Cosmic Ray Accelerators from Classical Electrodynamics
}

\author{
F.A. Aharonian ${ }^{1}$, A.A. Belyanin ${ }^{2,3}$, E.V. Derishev ${ }^{1,3}$, V.V. Kocharovsky ${ }^{2,3}$, Vl.V. Kocharovsky ${ }^{3}$ \\ ${ }^{1}$ MPI für Kernphysik, Saupfercheckweg 1, D-69117 Heidelberg, Germany \\ ${ }^{2}$ Dept. of Physics, Texas A\&M University, College Station, TX 77843-4242, USA \\ ${ }^{3}$ Institute of Applied Physics RAS, 46 Ulyanov st., 603950 Nizhny Novgorod, Russia
}

\begin{abstract}
We find the general requirements, set by classical electrodynamics, to the sources of extremely high-energy cosmic rays (EHECRs). It is shown that the parameters of EHECR accelerators are strongly limited not only by the particle confinement in large-scale magnetic field or by the difference in electric potentials (generalized Hillas criterion), but also by the synchrotron radiation, the electro-bremsstrahlung, or the curvature radiation of accelerated particles. Optimization of these requirements in terms of accelerator's size and magnetic field strength results in the ultimate lower limit to the overall source energy, which scales as the fifth power of attainable particle energy. Hard $\gamma$-rays accompanying generation of EHECRs can be used as a probe for potential acceleration sites. We apply the results to several populations of astrophysical objects - potential EHECR sources and discuss their ability to accelerate protons to $10^{20} \mathrm{eV}$ and beyond. A possibility to gain from ultrarelativistic bulk flows is emphasized, with Active Galactic Nuclei and Gamma-Ray Bursts being the examples.
\end{abstract}

PACS numbers: 98.70.Sa, 95.30.Gv

\section{INTRODUCTION}

The existence of EHECRs with energies above $10^{20} \mathrm{eV}$ is now a well-established fact (see, e.g. [1] and [2] for a review). Nearly perfect isotropy of the highest energy cosmic rays points to their likely extragalactic origin. On the other hand, the sources must be located within $100 \mathrm{Mpc}$ : the propagation distance for EHECRs of energy greater than $5 \times 10^{19} \mathrm{eV}$ is limited due to their interaction with the microwave background radiation [3, 4], unless there are some yet unknown type(s) of particles that do not undergo such an interaction.

The presence of non-thermal particles is a usual feature of any plasma system. However, finding a way to accelerate these particles to $10^{20} \mathrm{eV}$ is a serious theoretical challenge. Even if an electrodynamical system used to accelerate charged particles is sufficiently longlived, the attainable energy is limited since the particles can only stay within the acceleration region until their gyroradius exceeds the size of the region. (This is known as Hillas criterion [5].) Various types of modeland source-dependent radiative and collisional losses decrease the limiting particle energy furthermore. But even if one finds a way to circumvent the latter obstacle, there are radiative losses caused by the same electromagnetic field that is used to accelerate particles. For example, Greisen [6] considered the synchrotron losses and showed that for very small-sized accelerators, i.e., for those having very strong magnetic field, the synchrotron radiation drains energy so fast that even a particle accelerated at the largest possible rate for the magnetic-field dominated environments, $q B c$ ( $q$ is the particle charge, $B$ the magnetic field strength, $c$ the velocity of light), is not able to achieve the energy given by the Hillas criterion. However, Greisen's limit might seem to be not a general one, as in some objects accelerated particles move along the magnetic field lines (neutron stars are a perfect example of that), without suffering from the synchrotron losses.

In this paper, we relax the requirements to the acceleration sites as much as possible, assuming that inhomogeneity of electric and magnetic fields is small enough not to cause particle diffusion out of the acceleration site and taking into account only the radiation mechanism which gives the lowest energy loss rate. In fact, even if the synchrotron losses (or the electro-bremsstrahlung in the case of electric-field dominated environments) tend to zero, i.e., for particles moving along smooth field lines, there is still curvature radiation, which then limits the maximal particle energy. Furthermore, we generalize the analysis introducing acceleration efficiency parameter $\eta$, so that the acceleration rate is given by

$$
\dot{\varepsilon}=\eta q B c
$$

where $\eta B=E_{\text {eff }}$ is the projection of an electric field on the particle trajectory, effectively averaged as the particle moves along this trajectory. Such a representation is useful when, e.g., diffusive shock acceleration scenario is brought into consideration.

We derive the ultimate lower limit for the total electromagnetic energy stored in an acceleration region and find the size and the field strength that are optimal with respect to minimization of this energy. Then we consider a possible gain from relativistic motion of an accelerator as a whole and discuss what kind of additional information one gets from analysis of hard $\gamma$-photons generated as a byproduct of cosmic ray acceleration. Finally, we make comparison of theoretical constraints with the parameters of known astrophysical objects. 


\section{ELECTRODYNAMICAL LIMITATIONS}

Let us consider a region of space of a size $R$ where particle acceleration occurs. A natural assumption is that there is nothing like linear accelerator geometry in this region, but instead the field lines of both electric and magnetic fields are curved with the curvature radius being of the order of $R$ or less.

If the magnetic field is much stronger than the electric field, $B \gg E$, then a test particle is accelerated following a magnetic field line in its trajectory. If $E \gtrsim B$ but $(\vec{E} \cdot \vec{B}) \sim E B$, then particle's trajectory depends on the radiative loss rate. High loss rate will force the particle to follow a field line (of an electric field in this case) since a transverse to the field line component of particle's momentum will quickly decay due to an increase in radiative losses. In the opposite case, the field geometry will have little effect on particle's trajectory which therefore will make an angle of $\sim 45^{\circ}$ with field lines. The remaining case, where both Lorentz invariants $(\vec{E} \cdot \vec{B})$ and $\left|E^{2}-B^{2}\right|$ are much smaller than $E^{2}+B^{2}$, can be reduced to one of the already mentioned situations by the appropriate Lorentz transformation to the reference frame in which the mean (after averaging in space and time) Poynting flux density becomes zero.

In what follows we assume that accelerator's lifetime is at least as large as the acceleration timescale and use the most optimistic premise that the strongest of magnetic and electric fields is regular on scales $\ll R$ and quasistatic. (Alternating field direction implies that particles escape more readily and that the time-averaged acceleration rate is smaller, while the radiative losses are roughly the same.) Then, the effect of small-scale magnetic field can be neglected if $E>B$, and in the case $B>E$ the factor $\eta<1$ takes into account all necessary averaging for a small-scale and/or variable electric field, so that $E_{\text {eff }}=\eta B$.

The particle can be accelerated up to the terminal energy which is the smallest of either the work done by accelerating force in a time it takes for a particle to escape from the region, or the energy at which radiative losses balance the acceleration. The balance condition requires that the radiative loss rate $\dot{\varepsilon}_{\text {rad }}$ is equal to the energy gain rate, i.e.

$$
\dot{\varepsilon}_{\mathrm{rad}}=\frac{2}{3} \gamma^{4} \frac{q^{2}}{R^{2}} c=\eta q B c
$$

in the case of curvature-radiation dominated losses [7] (the particle is assumed to move along an arc of radius $R)$, or

$$
\dot{\varepsilon}_{\mathrm{rad}}=\frac{2}{3} \gamma^{2}\left(\frac{q^{2}}{m c^{2}}\right)^{2} c\left(B_{\perp}^{2}+E_{\perp}^{2}\right)=\eta q B c
$$

in the case of synchrotron- or electro-bremsstrahlung dominated losses. Here $\gamma$ is the Lorentz factor of the particle, $m$ its mass, $B_{\perp}$ and $E_{\perp}$ are the r.m.s. field components perpendicular to the particle momentum.
The comparison of Eqs. (2) and (3) shows that the curvature radiation gives a more favorable (smaller) estimate for the radiative losses as long as the particle energy, $\varepsilon=\gamma m c^{2}$, satisfies the condition

$$
\varepsilon<q R \sqrt{B_{\perp}^{2}+E_{\perp}^{2}} .
$$

If the above condition were violated, it would mean one of the following. Either the particle is no longer bound in the acceleration region, i.e., its gyro-radius $r_{\mathrm{H}}=\varepsilon /\left(q B_{\perp}\right)$ is larger than $R$ (when $r_{\mathrm{H}} \gtrsim R$, the very idea of curvature radiation becomes meaningless) and acceleration terminates upon the particle escape at $\varepsilon \leq q R B_{\perp}$, or, if $B_{\perp} \ll E_{\perp}$, the particle is unbound from the very beginning and its energy gain is limited by the difference of electric potentials across the acceleration region, $\varepsilon \leq q R E_{\text {eff }}=\eta q R B$. This provides a generalized version of the Hillas criterion.

Hereafter we neglect numerical factors of $\sim 1$ assuming $B=B_{\perp}$ and $E=E_{\perp}=E_{\text {eff }}=\eta B$. In general, one should bear in mind that for an inhomogeneous smallscale field the r.m.s. quantity $E^{2}$ must be replaced with $\left\langle E^{2}\right\rangle=E^{2} / \delta$, where $\delta<1$ is a duty-factor. (The same is true for magnetic field, although the situations where $E>B$ and the magnetic field has the duty-factor $\delta \ll 1$ seem quite unrealistic.) We assume below $\delta=1$, aiming to the most efficient acceleration. Thus, the condition given by Eq. (4) cannot be violated, as it satisfies the generalized Hillas criterion,

$$
\varepsilon_{\max }<q R \max \{B, E\} .
$$

Since we are interested in the most efficient acceleration (i.e., the highest acceleration rate at smallest radiative losses), it is Eq. (2) that will be used below to put the lower limit on the radiative loss rate. Then, we conclude that the maximal energy of a particle is either determined by the balance condition given by Eq. (2),

$$
\varepsilon_{\max }^{4}=\frac{3 \eta B R^{2}}{2 q}\left(m c^{2}\right)^{4},
$$

or is limited by the Eq. (5), whichever gives the smaller value.

The overall energy of an electromagnetic field in a spherical region of radius $R$, capable of accelerating particles up to $\varepsilon_{\max }$, is $W_{\mathrm{em}}=R^{3}\left(B^{2}+E^{2}\right) / 6$. According to Eqs. (5) and (5), respectively, it is limited as follows:

$$
W_{\mathrm{em}}>\frac{2}{27} \frac{q^{2}}{R}\left(\frac{\varepsilon_{\mathrm{max}}}{m c^{2}}\right)^{8} \frac{1+\eta^{2}}{\eta^{2}}
$$

and

$$
W_{\mathrm{em}}>\frac{R}{6}\left(\frac{\varepsilon_{\max }}{q}\right)^{2},
$$

Our interest is to lower the energy requirements taking $R$ as large as possible in Eq. (7a) and as small as possible 


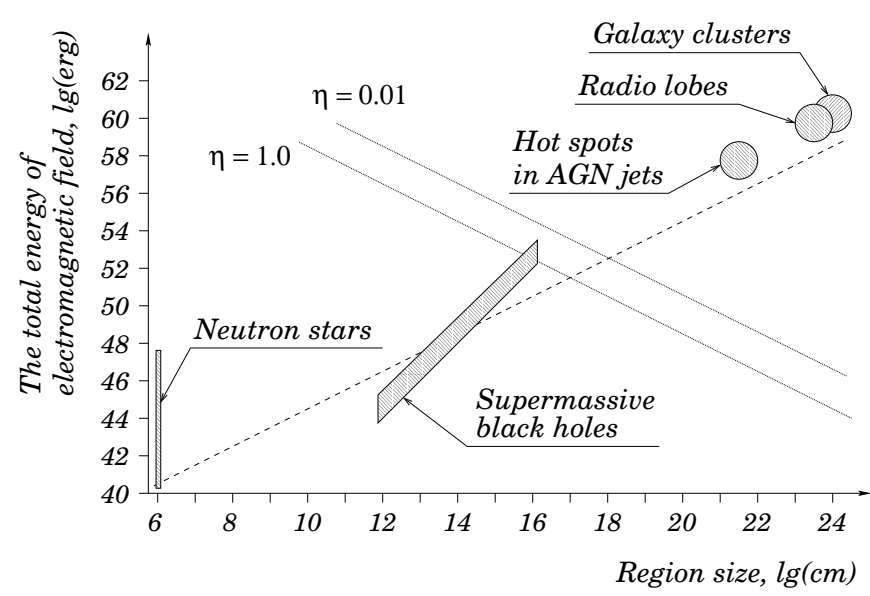

FIG. 1: The energy requirements for protons of energy $10^{20} \mathrm{eV}$. Eq. (月a), dotted lines (for acceleration efficiencies $\eta=1$ and $\eta=10^{-2}$ ), and Eq. ( $(1 \mathrm{~b})$, dashed line, as functions of the size of an acceleration region.

in Eq. (7b). Doing this, we derive the optimal size

$$
R^{(\mathrm{opt})} \simeq \frac{2}{3} \frac{\sqrt{1+\eta^{2}}}{\eta} \frac{q^{2} \varepsilon_{\max }^{3}}{\left(m c^{2}\right)^{4}}
$$

such that both conditions become quantitatively the same. This finally gives the optimal (the minimum possible) estimate for the amount of electromagnetic energy stored in the acceleration region:

$$
W_{\mathrm{em}}^{(\mathrm{opt})} \simeq \frac{1}{9} \frac{\sqrt{1+\eta^{2}}}{\eta} \frac{\varepsilon_{\max }^{5}}{\left(m c^{2}\right)^{4}} .
$$

The corresponding optimal strength of the magnetic field is

$$
B^{(\mathrm{opt})} \simeq \frac{3}{2} \frac{\eta}{1+\eta^{2}} \frac{\left(m c^{2}\right)^{4}}{q^{3} \varepsilon_{\max }^{2}}
$$

and $E^{(\mathrm{opt})}=\eta B^{(\mathrm{opt})}$.

The energy requirements given by Eqs. (7) as functions of accelerator size are presented in Fig. 1. The required accelerator energy increases as the region size deviates from the optimal one. A smaller region would accelerate particles to the energy $\varepsilon_{\max }$ only if the energy budget is increased by $R^{(\mathrm{opt})} / R$ times; in a larger region, the energy budget has to be increased by $R / R^{(\mathrm{opt})}$ times.

To have an idea of typical numbers, let us make the estimates for a proton of energy $\varepsilon_{\max }=10^{20} \mathrm{eV}$. In this case, the total electromagnetic energy in the acceleration region should exceed $W_{\mathrm{em}}^{(\mathrm{opt})} \simeq 3 \times 10^{51} \mathrm{erg}$; the optimal size $R^{(\mathrm{opt})}$ is of the order of $10^{17} \mathrm{~cm}$ and the optimal magnetic field is of $\sim 3 \mathrm{G}$. When making these estimates we assumed $\eta=1$ to lower the energy requirements. Taking $\eta$ still larger does not reduce them too much, by a factor of 2 at most.

All the results obtained are valid also for an accelerator that moves as a whole with the Lorentz factor $\Gamma \gg 1$,

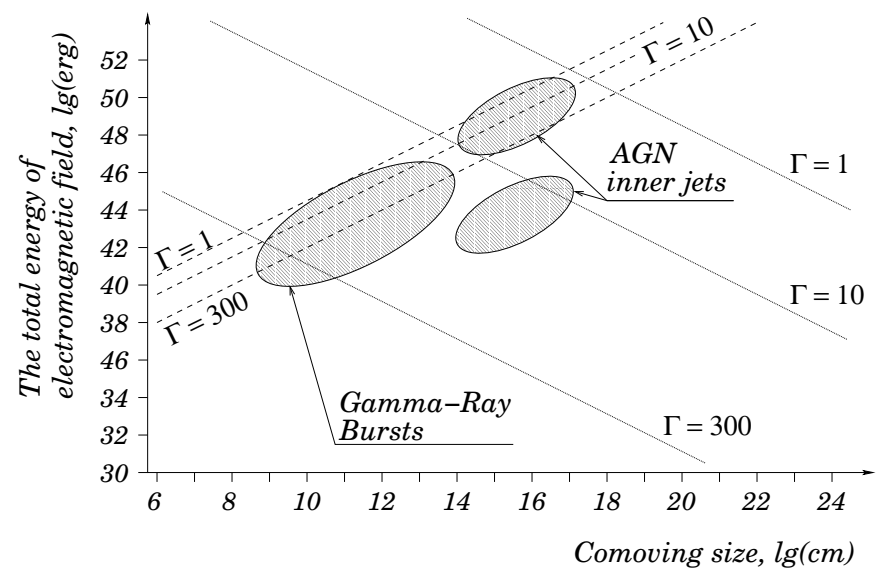

FIG. 2: The energy requirements for protons of energy $10^{20} \mathrm{eV}$. Eq. (7a), dotted lines (for accelerator at rest and for bulk Lorentz factors $\Gamma=10$ and $\Gamma=300)$, and Eq. $(7 \mathrm{~b})$, dashed lines, as functions of the comoving size of an acceleration region. For AGN inner jets, the upper zone corresponds to hadronic models of high-energy $\gamma$-ray emission, and lower zone - to leptonic models.

if the quantities are measured in the comoving frame. However, it is more convenient to present the results in a form where $W_{\mathrm{em}}^{(\mathrm{opt})}$ and $\varepsilon_{\max }$ are measured in the laboratory frame, while $R^{(\mathrm{opt})}, B^{(\mathrm{opt})}$ and $E^{(\mathrm{opt})}$ are measured in the comoving frame. Then

$$
\begin{aligned}
W_{\mathrm{em}}^{(\mathrm{opt})} & \simeq \frac{1}{9 \Gamma^{4}} \frac{\sqrt{1+\eta^{2}}}{\eta} \frac{\varepsilon_{\max }^{5}}{\left(m c^{2}\right)^{4}}, \\
R^{\prime(\mathrm{opt})} & \simeq \frac{2}{3 \Gamma^{3}} \frac{\sqrt{1+\eta^{2}}}{\eta} \frac{q^{2} \varepsilon_{\max }^{3}}{\left(m c^{2}\right)^{4}}, \\
B^{\prime(\mathrm{opt})} & \simeq \frac{3 \Gamma^{2}}{2} \frac{\eta}{1+\eta^{2}} \frac{\left(m c^{2}\right)^{4}}{q^{3} \varepsilon_{\max }^{2}},
\end{aligned}
$$

where the primed quantities are measured in the comoving frame. A comparison of energy requirements given by Eqs. (7), generalized for the case $\Gamma \gg 1$, for accelerators with different bulk Lorentz factors is presented in Fig. 2.

Apparently, the acceleration of EHECRs in ultrarelativistic bulk flows has an advantage of bringing the optimal source size to a more "comfortable" (for short-lived phenomena like Gamma-Ray Bursts) range and reducing the energy requirements to the source. However, for wind-like relativistic flows the actual requirements are geometry-dependent. Indeed, for the causality reasons, the acceleration region does not occupy the whole sphere of radius $R$, but rather extends to a distance $R^{\prime}=R / \Gamma$ transverse to the radius and to a distance $R^{\prime} / \Gamma$ along it, so that the total energy within the volume of radius $R$ is of $\sim \Gamma^{4} W_{\mathrm{em}}$ for a wind with a broad beam pattern. Since the energy stored in the acceleration region, $W_{\mathrm{em}}$, is multiplied by $\Gamma^{4}$ in this case, one can only gain from 
a more favorable ratio $R^{\prime} / R^{\prime \text { (opt) }}$ (cf. Eq. (11a)). A jet geometry is more favorable: with the jet opening angle $\simeq 1 / \Gamma$ the energy requirements can be reduced by a factor as large as $\Gamma^{2}$, with respect to the non-relativistic accelerator. To implement the advantage of relativistic bulk motion to the full extent, i.e., to get a gain by a factor of $\Gamma^{4}$, one needs a transient jet source which lasts for a time $\simeq R /\left(\Gamma^{2} c\right)$.

\section{CONSTRAINTS DUE TO RADIATION FROM ACCELERATION SITES}

Before moving to an overview of different source populations, let us discuss some implications regarding the acceleration timescale and the electromagnetic radiation accompanying the acceleration process.

When $\eta \gtrsim 1$, a particle is accelerated up to $\varepsilon_{\max }$ in a time $t_{\text {acc }} \lesssim R / c$, which is less than or comparable to the dynamical timescale of an accelerator. However, at low efficiency $\eta \ll 1$, the acceleration may take a longer time, i.e. $\eta^{-1}(R / c)$, if accelerator's size is larger than $R^{\text {(opt) }}$, or $\eta^{-1}\left(R / R^{(\mathrm{opt})}\right)(R / c)$ otherwise. Relativistic motion of an accelerator usually implies relativistic expansion. Consequently, the lifetime is of order $R / c$, so that small $\eta$ strongly disfavors such objects unless their size is $\lesssim \eta R^{(\mathrm{opt})}$, that would allow a particle to attain the energy $\varepsilon_{\max }$.

The acceleration of particles goes side by side with the emission of hard electromagnetic radiation. The lower limit on the energy lost to this radiation is given by the product of $t_{\text {acc }}$ and the radiative loss rate (Eq. (2), where it is convenient to substitute $\gamma^{3}$ from Eq. (8)). However, if $t_{\text {acc }}<R / c$ one must integrate the loss rate over a time of order $R / c$, since a particle cannot leave the acceleration region in a shorter time. As a result of particle's acceleration, the following fraction of its terminal energy $\varepsilon_{\max }$ is lost for $\gamma$-radiation

$$
\frac{E_{\mathrm{rad}}}{\varepsilon_{\max }} \gtrsim \begin{cases}R^{(\mathrm{opt})} / R & \text { if } R>R^{(\mathrm{opt})} \\ 1 & \text { if } R^{(\mathrm{opt})}>R>\eta R^{(\mathrm{opt})} \\ \eta R^{(\mathrm{opt})} / \sqrt{1+\eta^{2}} R & \text { if } R<\eta R^{(\mathrm{opt})} .\end{cases}
$$

Therefore, acceleration in small objects $\left(R<R^{(\mathrm{opt})}\right)$ is always limited by radiative losses.

The spectral energy distribution of the accompanying radiation has a maximum (in the source frame) around

$$
\varepsilon_{\gamma} \sim \hbar \frac{\gamma^{3} c}{R} \sim \eta \frac{R^{(\mathrm{opt})}}{R} \frac{m c^{2}}{\alpha Z^{2}},
$$

provided the accelerator size is less than $R^{(\mathrm{opt})} ; \alpha$ is the fine-structure constant and $Z$ the ion charge in units of the elementary charge. The energy of curvature radiation photons given by Eq. (13) is related to the energy of synchrotron photons as $\varepsilon_{\gamma}=\left(r_{\mathrm{H}} / R\right) \varepsilon_{\gamma}^{\mathrm{sy}}$.

The ratio of the luminosity in accompanying radiation to the luminosity in accelerated particles, $L_{\gamma} / L_{\text {acc }}$, as well as the energy of individual photons, is reduced if the acceleration time is limited to $R / c$, like in the sources with relativistic bulk motion (particle diffusion may lead to analogous limit), but this is at expense of reducing $\varepsilon_{\max }$. Since the radiative loss rate (Eq. (2)) and the energy of individual photons (Eq. (13)) scale as $\gamma^{4}$ and $\gamma^{3}$, while particle's Lorentz factor increases linearly with time, the corresponding factors for $L_{\gamma} / L_{\mathrm{acc}}, \varepsilon_{\gamma}$ and $\varepsilon_{\max }$ are: $\left(\eta R^{(\mathrm{opt})} / R\right)^{4},\left(\eta R^{(\mathrm{opt})} / R\right)^{3}$, and $\left(\eta R^{(\mathrm{opt})} / R\right)$ respectively, in the case where $R^{(\mathrm{opt})}>R>\eta R^{(\mathrm{opt})}$. Note that if the maximal energy were limited by the synchrotron rather than curvature losses, then the acceleration up to $\varepsilon_{\max }$ would not be possible at all in the objects smaller than $R^{(\mathrm{opt})}$ if $\eta \leq 1$, as noted in 6 .

The check whether the sky-averaged luminosity in accompanying electromagnetic emission exceeds the diffuse background is a powerful selection tool for possible acceleration sites. For example, the acceleration of protons with high efficiency, $\eta \simeq 1$, results in $\gamma$-radiation in energy range $\gtrsim 100 \mathrm{GeV}$ (Eq. (13)). Such photons undergo reprocessing into $10 \mathrm{MeV}$ - $100 \mathrm{GeV}$ energy range through interaction with cosmic infrared and microwave backgrounds. The observed spectral energy distribution of diffuse $\gamma$-ray background, $\sim 10^{-9} \mathrm{erg} /\left(\mathrm{cm}^{2} \mathrm{~s} \mathrm{sr}\right)^{-1}$ [8], is virtually constant in the $10 \mathrm{MeV}-100 \mathrm{GeV}$ spectral domain, and the observed flux of $10^{20} \mathrm{eV}$ cosmic rays is $\sim 2-5 \times 10^{-12} \mathrm{erg} /\left(\mathrm{cm}^{2} \mathrm{~s} \mathrm{sr}\right)^{-1}$ [2], i.e., $200-500$ times less. But the GZK effect limits the distance to which the sources of $10^{20} \mathrm{eV}$ cosmic rays can be observed to $\sim 30 \mathrm{Mpc}$, while the related $\gamma$-radiation is visible up to a redshift $z \sim 1$, i.e. the distance $\simeq 3 \mathrm{Gpc}$. If the accelerators are uniformly distributed in the Universe and no other contributions to the $\gamma$-ray background are taken into account, this implies $L_{\gamma} / L_{\text {acc }} \lesssim 5$ and hence $R /\left(\eta R^{(\mathrm{opt})}\right) \gtrsim 1 / 5$. Since the spectral energy distribution of the $\gamma$-ray background is nearly constant in $10 \mathrm{MeV}-100 \mathrm{GeV}$ range, the same limit applies for iron nuclei, although their individual $\gamma$-luminosity is concentrated in the lower-energy spectral domain $\gtrsim 10 \mathrm{GeV}$ (see Eq. (13), which gives scaling $\varepsilon_{\gamma} \propto A / Z^{2}$ ).

The accompanying radiation may also help in identifying acceleration sites. The electrons are more suitable for this purpose as they can achieve radiative-loss limited regime of acceleration in an object with much smaller energy content. For example, there is an excess emission in $100 \mathrm{MeV}$ range from the Crab plerion, which was interpreted as the electron synchrotron radiation [9]. Interestingly, the size of the plerion, $\sim 10^{17} \mathrm{~cm}$, and its estimated magnetic field, $\sim 10^{-4} \mathrm{G}$, are very close to the optimal values for electrons accelerated at the efficiency $\eta=1$. The electrons then attain the energy $\simeq 3 \times 10^{15} \mathrm{eV}$ and the peak of their synchrotron spectrum is at $\simeq 100 \mathrm{MeV}$.

So far, we did not take into account the proton interaction with smallest-scale electromagnetic field, which results in photomeson reactions (at wavelengths $<0.04 \mathrm{~cm}$ or photon energies $>2 \times 10^{-3} \mathrm{eV}$ for $10^{20} \mathrm{eV}$ proton). Such an interaction, with the cross-section $\sigma_{p \gamma} \simeq$ $2 \times 10^{-28} \mathrm{~cm}^{2}$ and inelasticity $f \simeq 0.5$, may in some cases 
lead to unacceptably high energy losses for the accelerated particles. For illustration, we make the estimate for $10^{20} \mathrm{eV}$ protons and for the most favorable case $\eta=1$. In order to allow accelerated protons to escape without significant losses, the luminosity of the source at the maximum of integral photon spectrum must be

$$
L<\pi \frac{R c}{f \sigma_{p \gamma}} \bar{\varepsilon} \simeq 1.5 \times 10^{44}\left(\frac{R}{10^{17} \mathrm{~cm}}\right)\left(\frac{\bar{\varepsilon}}{1 \mathrm{eV}}\right) \mathrm{erg} / \mathrm{s},
$$

where $\bar{\varepsilon}$ is the location of the maximum.

\section{OVERVIEW OF POSSIBLE ACCELERATION SITES}

Neutron stars. Because of their size, which is far too small compared to $R^{(\mathrm{opt})}$, the performance of neutron stars as cosmic ray accelerators is limited be the curvature losses (see, e.g., [7]). For $10^{20} \mathrm{eV}$ EHECRs, the energy requirements to neutron star magnetospheres are unphysical: $\sim 10^{62} \mathrm{erg}$ for protons and $\sim 10^{51} \mathrm{erg}$ for iron nuclei. Acceleration of heavier nuclei, with mass $A \sim 150$ and charge $Z \sim 50$, to slightly smaller energy $5 \times 10^{19} \mathrm{eV}$ is marginally possible for rapidly rotating (i.e. $\eta \sim 1$ ) neutron stars with magnetic field $B \sim 10^{15} \mathrm{G}$ (so-called magnetars). However, the question whether such nuclei can be efficiently transported to neutron star surface and ejected from it remains open. In addition, the luminosity in accompanying $10-100 \mathrm{TeV}$ curvature $\gamma$-radiation (which is likely to be reprocessed through electromagnetic cascade into $1-100 \mathrm{MeV}$ range) would be 5 to 6 orders of magnitude higher than in the produced EHECRs. Particles accelerated near the light cylinder produce less accompanying radiation, but the energy requirements rise in this case. Therefore, magnetospheres of neutron stars have to be excluded from the list of EHECR sources.

A way out from the problem of catastrophic overproduction of the accompanying radiation is acceleration of particles within ultrarelativistic pulsar winds. However, the energy constraints, which now have to be reformulated in terms of required Poynting-flux luminosity $L_{\mathrm{em}} \sim \Gamma^{4} W_{\mathrm{em}} c / R$ for a wind with a broad beam pattern, remain extremely tough. The best case $\left(R^{\prime}=R^{\prime(\mathrm{opt})}\right.$ and $\eta=1$ ) for $10^{20} \mathrm{eV}$ protons corresponds to $\Gamma \lesssim 600$ and $L_{\mathrm{em}} \simeq 10^{45} \Gamma^{2} \mathrm{erg} / \mathrm{s}$. With iron nuclei the figures become $\Gamma \lesssim 60$ and $L_{\mathrm{em}} \simeq 1.5 \cdot 10^{42} \Gamma^{2} \mathrm{erg} / \mathrm{s}$. In any case, the required luminosity is far beyond any of the observed values.

Black holes. Rapidly rotating black holes embedded in a magnetic field can generate electric field and accelerate particles (e.g., [10, 11]). We make an (optimistic) estimate assuming that the magnetic field is roughly in equipartition with accreted plasma, an accretion rate corresponds to the Eddington regime, and acceleration occurs within $3 R_{g}$, where $R_{g} \simeq 3 \times 10^{5} \mathrm{~cm} M / M_{\odot}$ is the Schwarzschild radius of the black hole of mass $M$.
The energy $W_{\mathrm{em}} \sim \pi m_{p} c^{2} R_{g}^{2} / \sigma_{T}$ is available in the acceleration region $\left(m_{p}\right.$ is the proton mass, and $\sigma_{T} \simeq$ $6.6 \times 10^{-24} \mathrm{~cm}^{2}$ the Thomson cross-section). This is to be compared with the required energy $\left(R^{(\mathrm{opt})} / 3 R_{g}\right) W_{\mathrm{em}}^{(\mathrm{opt})}$, which yields

$$
\begin{aligned}
R_{g} & >\left(\frac{2}{81 \pi} \sigma_{T} \frac{1+\eta^{2}}{\eta^{2}} \frac{q^{2} \varepsilon_{\max }^{8}}{\left(m c^{2}\right)^{8} m_{p} c^{2}}\right)^{1 / 3} \\
& \simeq 3 \times 10^{15} Z^{2 / 3} A^{-8 / 3} \mathrm{~cm} .
\end{aligned}
$$

If the accelerated particles are protons, then only supermassive black holes with $M>10^{10} M_{\odot}$ can meet the above requirement. On the other hand, a lighter hole of $M>2 \times 10^{6} M_{\odot}$ can accelerate iron nuclei, and, since the size of such a hole is close to the optimal one or exceeds it $\left(R_{g} \gtrsim R^{(\mathrm{opt})} \simeq 8 \times 10^{12} \mathrm{~cm}\right)$, this would not be at odds with the observed $\gamma$-ray background.

Active Galactic Nuclei (AGNs). This phenomenon is due to ejection of a relativistic jet by a massive black hole interacting with an accretion disk around it. Inner jets in AGNs have typically Lorentz factors between 5 and 10 . The estimate of the magnetic field depend on the radiation model. For leptonic models which imply high-energy $\gamma$-ray production through inverse Compton scattering, the magnetic field is of $\sim 0.1 \mathrm{G}$ (e.g., [12]). However, in hadronic models, in particular in the extreme proton-synchrotron model, the field strength exceeds 100 G 113]. Furthermore, the size of acceleration region, $10^{14}-10^{16} \mathrm{~cm}$ as inferred from variability timescale, is close to the optimal value for $10^{20} \mathrm{eV}$ protons. This eliminates problems with $\gamma$-ray background and minimizes energy requirements that constrain the Poynting-flux luminosity of the source in the case of a relativistic outflow. This luminosity $\left(\Gamma W_{\mathrm{em}} c / R^{\prime}\right)$ must be higher than $\sim 10^{45} \mathrm{erg} / \mathrm{s}$ (or a factor of $(\theta \Gamma)^{2}$ more if the opening angle of the jet, $\theta$, is larger than $\Gamma^{-1}$ ), which is reasonable for a typical AGN.

Large accelerators. There are three kinds of very large objects that were proposed to produce EHECRs: knots (or hot spots) in large-scale AGN jets, radio lobes (shocked regions), and galaxy clusters (e.g., [5, 14, 15). Despite their sizes are far from the optimum: $\sim 1 \mathrm{kpc}$, $\sim 100 \mathrm{kpc}$, and $\sim 1 \mathrm{Mpc}$ respectively, the energy of a magnetic field is enough to bind $10^{20} \mathrm{eV}$ protons. The limits on these objects are better formulated in terms of the requirements to the acceleration efficiency $\eta$, and they are likely to arise from particle diffusion. In all cases there is about 1000-fold excess of the energy stored in magnetic field with respect to that shown in Fig. 1. Therefore, if particles escape on the Bohmdiffusion timescale $\sim 3 R^{2} /\left(r_{H} c\right)$, a rather high acceleration efficiency $\eta \gtrsim 0.03$ is necessary. Even if the diffusion is strongly suppressed (e.g., special configuration of magnetic field lines), there are limits on $\eta$. It must be larger than $\sim(0.3-1) \times 10^{-2}$ in radio lobes and galaxy clusters, otherwise protons are accelerated so slowly that they start to lose energy interacting with the cosmic microwave background, which eventually stops acceleration. 
In hot spots, with their stronger magnetic field up to $B \sim 10^{-3} \mathrm{G}, \eta$ can be as small as few $\times 10^{-5}$, in which case their size becomes slightly smaller than the optimal one leading to a further increase in energy requirements.

Gamma-Ray Bursts (GRBs). These explosionlike events have been argued to be suitable places for acceleration of EHECRs (e.g., [16, 17]), owing to their compactness (variability timescale less than a second), high apparent $(4 \pi)$ luminosity of the order of $10^{52} \mathrm{erg} / \mathrm{s}$, and ultrarelativistic bulk flows with the Lorentz factors $\Gamma \sim 100-1000$, which are the key ingredients of any GRB model (see, e.g., 18 for a review). Acceleration may take place either in internal shocks formed within the outflow (at a distance $10^{10}-10^{13} \mathrm{~cm}$ from the central engine) by colliding shells of different Lorentz factors or in the external shock (at a distance $10^{16}-10^{17} \mathrm{~cm}$ ) at the interface between ejected material and interstellar gas. The distance from which the accelerated protons can escape is, however, limited at least by their interaction with the intense thermal photon field left after adiabatic expansion of relativistic fireball. The escape distance is (in the shock comoving frame)

$$
R_{\mathrm{esc}}^{\prime} \simeq 0.08 \frac{f \sigma_{p \gamma} L}{\pi \Gamma^{3} c T} \simeq \frac{2 \times 10^{17} \mathrm{~cm}}{\Gamma^{3}} \frac{L}{10^{52} \mathrm{erg} / \mathrm{s}} \frac{3 \mathrm{MeV}}{T},
$$

where $L$ is the GRB $4 \pi$-luminosity and $T$ the temperature at the base of the fireball, $T \simeq 3 \mathrm{MeV}$. The Eqs. (11b) and (16) scale with the same power of $\Gamma$, so that the ratio between $R_{\mathrm{esc}}^{\prime}$ and $R^{\prime(\mathrm{opt})}$ depends practically only on GRB luminosity, and the escape distance appears to be larger than the optimal size, unless a GRB has unusually low luminosity. Therefore, radiative losses are negligible and the maximal particle energy is $\varepsilon_{\max }^{\prime}=\eta q B^{\prime} c\left(R^{\prime} / c\right)$. With the magnetic field related to the GRB $4 \pi$-luminosity via the parameter $\epsilon_{m}$, which is the fraction of energy carried in the form of magnetic field, this gives

$$
\varepsilon_{\max }=\Gamma \varepsilon_{\max }^{\prime}=\frac{\eta \epsilon_{m}^{1 / 2}}{\Gamma} q \sqrt{\frac{2 L}{c}} \sim \frac{2.5 \times 10^{23} \mathrm{eV}}{\Gamma} .
$$

Note that the maximal energy $\varepsilon_{\max }$ decreases with increasing bulk Lorentz factor $\Gamma$. This can be understood following the same reasoning as at the end of the Sect. II: changing the Lorentz factor allows one to gain only from a more favorable ratio $R^{\prime(\mathrm{opt})} / R^{\prime}$, but for GRBs the escape distance is larger than the optimal size, so that the increase in $\Gamma$ makes things worse.

Since explanation of some GRB properties, such as rapid variability and the presence of emission in the $10 \mathrm{GeV}$ domain 19], requires $\Gamma>100$, we conclude that GRBs might be capable of accelerating protons well above $10^{20} \mathrm{eV}$. However, to match the observed cosmic ray flux at least 50 per cent of the observed GRB energy has to be converted into EHECRs.

\section{CONCLUSION}

Radiation losses, either due to synchrotron or curvature radiation, play increasing role in the energy balance of accelerated particles with decreasing size (and accordingly increasing magnetic field) of the accelerator. In sufficiently compact objects the radiative losses limit maximal particle energy to the values that are smaller than those determined from the Hillas criterion. Thus, there are optimal size and magnetic field strength that minimize the requirements to the internal energy of the accelerator. For $10^{20} \mathrm{eV}$ protons and acceleration efficiency $\eta=1$, the optimal size is $R^{(\mathrm{opt})} \simeq 10^{17} \mathrm{~cm}$ and the optimal magnetic field is $B^{(\mathrm{opt})} \simeq 3 \mathrm{G}$. This corresponds to the total electromagnetic energy in the acceleration region $W_{\mathrm{em}}^{(\mathrm{opt})} \simeq 3 \times 10^{51}$ erg. Relativistic bulk motion reduces the energy requirements and the optimal comoving size, while increasing the optimal comoving magnetic field. The Lorentz factor $\Gamma=10$ leads to $R^{\prime(\mathrm{opt})} \simeq 10^{14} \mathrm{~cm}$ and $B^{\prime(\mathrm{opt})} \simeq 300 \mathrm{G}$, corresponding to the total electromagnetic energy $W_{\mathrm{em}}^{(\mathrm{opt})} \simeq 3 \times 10^{47} \mathrm{erg}$ in the region of size $R^{\prime(\mathrm{opt})}$.

Efficient acceleration in optimally-sized objects leads to significant radiative losses, which in this case occur in a regime transitional between the synchrotron and the curvature radiation. The emission is concentrated at photon energies of $\sim 100-300 \mathrm{GeV}$ (protons) and $\sim 50-150 \mathrm{MeV}$ (electrons) times the bulk Lorentz factor. This suggests the use of new generation of air-Cherenkov telescopes and GLAST, respectively, for a search for efficient accelerators.

The derived constraints seem to firmly exclude any known population of neutron stars as acceleration sites of EHECRs. Black holes also appear to be not able to accelerate protons to $10^{20} \mathrm{eV}$ except for the supermassive ones with masses $M>10^{10} M_{\odot}$, although acceleration of heavy nuclei by lighter holes is still a possibility. Other astrophysical objects considered above are able, in principle, to accelerate EHECRs, although the required efficiency $\eta$ is always comparable to unity. In the standard treatment of the problem, such an efficiency is difficult to achieve even in ultrarelativistic shocks 20. In addition, energy losses due to photomeson reactions may be a serious problem for radiation-rich environments of supermassive black holes and for GRB sources.

The results obtained in this paper are the unavoidable limits, set by the laws of classical electrodynamics. Taking into account the effects of diffusion, the energy losses via photomeson process, the energy content of particles and waves in cosmic plasma, etc., should lead to still stronger limits. However, they cannot, of course, constrain the top-down models for the origin of EHECRs (e.g., 21]), which make use of quantum effects and nonelectromagnetic interactions.

In view of the development of advanced experiments which aim at detection of cosmic rays (CRs) above $10^{21} \mathrm{eV}$, it is worth noting what is the highest energy of 
EHECRs still consistent with the acceleration scenario of their origin. Plotting diagrams similar to Figs. 1 and 2, but for different values of $\varepsilon_{\max }$, one can see that in GRBs and AGN inner jets protons can in principle be accelerated up to $10^{21} \mathrm{eV}$. At such energies, acceleration is likely to be radiative-loss limited in both cases. Above $10^{21} \mathrm{eV}$ GRBs fail because of their insufficient duration and AGN inner jets - because of insufficient Poynting-flux luminosity. Hot spots, radio lobes and galaxy clusters can still work to $(3-5) \times 10^{21} \mathrm{eV}$ under very speculative assumption that the magnetic field is ordered on all scales and the acceleration efficiency is $\eta \simeq 1$. In this case, acceleration is escape-limited. At the energies $\gtrsim 10^{22} \mathrm{eV}$ the CR primaries have to be heavy nuclei. In all the sources listed above the heavy nuclei are accelerated in the escape-limited regime, so that the attainable energy is roughly $Z$ times more than for protons. However, the nuclei of such energy are fragmented through interaction with the microwave background photons after traveling a distance of less than $1 \mathrm{Mpc}$, that means they must be produced within the local group of galaxies, and GRBs is the only possibility to do this. Although the nuclei are easily fragmented in radiation-reach environments of GRBs, we have to conclude that formally the primaries with energy up to $(2-3) \times 10^{22} \mathrm{eV}$ can be produced within the framework of acceleration scenario.
[1] J.W. Cronin, Rev. Mod. Phys. 71, S165 (1999).

[2] M. Nagano and A. A. Watson, Rev. Mod. Phys. 72, 689 (2000).

[3] K. Greisen, Phys. Rev. Lett. 16, 748 (1966).

[4] G.T. Zatsepin, V.A. Kuzmin, Pis'ma Zh. Eksp. Teor. Fiz. 4, 114 (1966) [JETP. Lett. 4, 78 (1966)].

[5] A.M. Hillas, Annu. Rev. Astron. Astrophys. 22, 425 (1984).

[6] K. Greisen, in Proceedings of the 9th International Cosmic Ray Conference (Institute of Physics, London, 1965), Vol. 2, p. 609.

[7] V.S. Berezinskii, S.V. Bulanov, V.A. Dogiel, V.S. Ptuskin, 1990, Astrophysics of Cosmic Rays, ed. V. L. Ginzburg (Amsterdam: North-Holland),

[8] P. Sreekumar et al., Astrophys. J. 494, 523 (1998).

[9] O.C. de Jager et al., Astrophys. J. 457, 253 (1996).

[10] E. Boldt, M. Loewenstein, Mon. Not. RAS 316, L29 (2000).
[11] A. Levinson, Phys. Rev. Lett. 85, 912 (2000).

[12] M.-H. Ulrich, L. Maraschi, C.M. Urry, Annu. Rev. Astron. Astrophys. 35, 445 (1997).

[13] F.A. Aharonian, New Astron. 5, 377 (2000).

[14] C.A. Norman, D.B. Melrose, A. Achterberg, Astrophys. J. 454, 60 (1995).

[15] P.L. Biermann, P.A. Strittmatter, Astrophys. J. 322, 643 (1987).

[16] E. Waxman, Phys. Rev. Lett. 75, 386 (1995).

[17] M. Vietri, Astrophys. J. 453, 883 (1995).

[18] T. Piran, Phys. Rep. 314, 575 (1999).

[19] K. Hurley, Nature 372, 652 (1994).

[20] A. Achterberg, Y.A. Gallant, J.G. Kirk, A.W. Guthmann, Mon. Not. RAS 328, 393 (2001).

[21] V. Berezinsky, Nucl. Phys. B (Proc. Suppl.) 81, 311 (2000). 